INPLASY

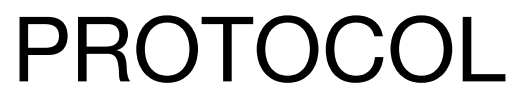

To cite: Zheng et al. Related factors for depression among Chinese men who have sex with men: A protocol for systematic review and metaanalysis. Inplasy protocol 2020110142. doi: 10.37766/inplasy2020.11.0142

Received: 29 November 2020

Published: 30 November 2020

Corresponding author: Jing Gao

gaojing@cdutcm.edu.cn

Author Affiliation:

Chengdu University of TCM

Support: None.

Review Stage at time of this submission: Preliminary searches.

Conflicts of interest: No conflict of interest.

\section{Related factors for depression among Chinese men who have sex with men: A protocol for systematic review and meta-analysis}

\author{
Zheng, YP1; Gao, J2; Jiang, XL³.
}

Review question / Objective: The aim of this study is to evaluate the related factors for depression among Chinese men who have sex with men.

Condition being studied: A number of studies have described various factors that influence or induce depression among men who have sex with men, and these factors reduce the mental health status of patients and even increase the risk of suicide. However, due to the influence of confounding factors such as study area, the conclusions of the study are not consistent. Therefore, the main purpose of this review is to evaluate the reported factors that contribute to depression among Chinese men who have sex with men.

INPLASY registration number: This protocol was registered with the International Platform of Registered Systematic Review and Meta-Analysis Protocols (INPLASY) on 30 November 2020 and was last updated on 30 November 2020 (registration number INPLASY2020110142).

\section{INTRODUCTION}

Review question / Objective: The aim of this study is to evaluate the related factors for depression among Chinese men who have sex with men.

Condition being studied: A number of studies have described various factors that influence or induce depression among men who have sex with men, and these factors reduce the mental health status of patients and even increase the risk of suicide. However, due to the influence of confounding factors such as study area, the conclusions of the study are not consistent. Therefore, the main purpose of this review is to evaluate the reported 
factors that contribute to depression among Chinese men who have sex with men.

\section{METHODS}

Search strategy: Studies of factors related to depression among Chinese men who have sex with men will be searched from CNKI, Wanfang Database, VIP Database, CBM, PubMed, Embase, Web of Science, and the Cochrane Library. The retrieval time is limited from the time of database construction to December 2020. And the search keywords are "men who have sex with men/MSM", "depression/depressive symptom/depressive disorder ", "risk factors/related factors/influence factors ", " China/Chinese". According to the types of different databases, this study adopts the search strategies by combining subject words with free words, and Boolean logic operators, truncation operators and so on are used for study search. Meanwhile, the references included in the study will be tracked and supplemented as much as possible.

Participant or population: Men who have sex with men in China.

Intervention: Related factors for depression among Chinese men who have sex with men.

Comparator: Chinese men who have sex with men without depression.

Study designs to be included: (1) Crosssectional studies; (2) case-control studies; (3) cohort studies.

Eligibility criteria: (1) inclusion criteria: 1) Types of studies: cross-sectional , casecontrol and cohort studies; 2) Types of participants: men who have sex with men in China; 3) Exposure factors: exposure factors are associated with depression among men who have sex with men, and the definition of each factor is the same or similar; 4) Outcome indicators: odds ratios (OR) values and $95 \%$ confidence intervals (CI) of related factors. (2) Exclusion criteria: 1) Duplicate publications and studies from the same data sources; 2) Studies that fail to obtain outcome indicators due to data missing and errors; 3) Reviews, commentaries, letters, case reports.

Information sources: Database retrieval will be conducted on CNKI, Wanfang Database, VIP Database, CBM, PubMed, Embase, Web of Science, and the Cochrane Library to search studies of related factors for depression among Chinese men who have sex with men published from the establishment of the database to December 2020.

Main outcome(s): The odds ratios (OR) values and $95 \%$ confidence intervals $(\mathrm{Cl})$ of related factors.

Quality assessment / Risk of bias analysis: Two researchers independently evaluate the quality of the studies, and in case of disagreement during the evaluation, the dispute will be resolved through discussion by two researchers or be submitted to a third research for resolution. NewcastleOttawa Scale (NOS) will be used for the risk assessment of bias in case-control studies and cohort studies. And the the bias risk assessment criteria of the Agency for Healthcare Research and Quality (AHRQ) that is used for the risk assessment of bias in cross-sectional studies.

Strategy of data synthesis: Stata $\mathbf{1 5 . 0}$ statistical software will be used for data analysis. The Cochran $Q$ test and 12 index will be applied to evaluate the heterogeneity of studies.

Subgroup analysis: If heterogeneity exists in the study, we will conduct a subgroup analysis based on age, region and etc.

Sensibility analysis: Sensitivity analyses will be performed by removing the included studies one by one in order to assess the changes of the overall effects after removing a certain study.

Language: English.

Country(ies) involved: China. 
Keywords: China; Men who have sex with men; Related factors; Meta-analysis.

Contributions of each author:

Author 1 - Yuping Zheng - The author's responsibilities are literature retrieval, data collection and analysis, risk of bias analysis and manuscript writing.

Author 2 - Jing Gao - The author participates in data analysis and manuscript revision.

Author 3 - Xiaolin Jiang - The author is involved in data extraction and risk of bias analysis. 